\title{
p300 mediates cellular resistance to doxorubicin in bladder cancer
}

\author{
ARIO TAKEUCHI ${ }^{1}$, MASAKI SHIOTA ${ }^{1}$, KATSUNORI TATSUGAMI $^{1}$, AKIRA YOKOMIZO $^{1}$, \\ SHINGO TANAKA ${ }^{1}$, KENTARO KUROIWA ${ }^{1}$, MASATOSHI ETO ${ }^{2}$ and SEIJI NAITO ${ }^{1}$ \\ ${ }^{1}$ Department of Urology, Graduate School of Medical Sciences, Kyushu University, Fukuoka 812-8582; \\ ${ }^{2}$ Department of Urology, Graduate School of Medical Sciences, Kumamoto University, Kumamoto 860-8556, Japan
}

Received July 5, 2011; Accepted September 6, 2011

DOI: $10.3892 / \mathrm{mmr} .2011 .593$

\begin{abstract}
Bladder cancer is one of the most common urogenital malignancies. At the non-invasive stage, bladder cancer can be completely resected transurethrally. However, $70 \%$ of patients experience intravesical tumor recurrence within 5 years. Patients with advanced bladder cancer frequently receive a chemotherapy regimen containing doxorubicin. However, doxorubicin resistance is a major obstacle to cancer chemotherapy. Previously, we reported that the histone acetyltransferase p300/CBP-associated factor is involved in doxorubicin resistance in bladder cancer. However, the role of another histone acetyltransferase, $\mathrm{p} 300$, in bladder cancer resistance to doxorubicin remains unclear. In this study, we investigated the molecular mechanism of doxorubicin resistance in bladder cancer with regard to p300. The result showed that p300 expression was reduced in doxorubicin-resistant bladder cancer cells and in response to doxorubicin exposure. Furthermore, p300 suppression rendered bladder cancer cells resistant to doxorubicin. Taken together, the results from this study indicate that p300 may be a promising molecular therapeutic target through the modulation of cellular sensitivity to doxorubicin in bladder cancer.
\end{abstract}

\section{Introduction}

Bladder cancer is one of the most common urogenital malignancies. At the non-invasive stage, bladder cancer can be completely resected transurethrally. However, $70 \%$ of patients experience intravesical tumor recurrence within 5 years. To prevent recurrence of non-invasive bladder cancer, the instillation of bacillus Calmette-Guérin, doxorubicin and mitomycin $\mathrm{C}$ has been empirically adapted, but these can suppress intravesical recurrence only modestly (1). However, anticancer agents, including doxorubicin, gemcitabine and cisplatin are administered for advanced bladder cancer (2).

Correspondence to: Dr Seiji Naito, Department of Urology, Graduate School of Medical Sciences, Kyushu University, Fukuoka 812-8582, Japan

E-mail: naito@uro.med.kyushu-u.ac.jp

Key words: bladder cancer, cisplatin, doxorubicin, p300
However, most bladder cancers recur with a terminal prognosis, even though these anticancer agents are empirically effective. Therefore, more effective intravesical and systemic chemotherapy for bladder cancer is required.

Doxorubicin has been administered for many types of solid tumors, including breast, hepatocellular and urothelial cancers. In bladder cancer, doxorubicin is employed as an agent of intravesical instillation into the bladder or by intravenous injection. Several molecules that are associated with the acquisition of doxorubicin resistance have been identified, including detoxifying enzymes, drug-efflux pumps and apoptosis-related genes (3). We have previously established doxorubicin-resistant bladder cancer cell lines $(4,5)$. However, the precise mechanism of doxorubicin resistance and the ability to overcome this resistance remains unresolved.

p300 was originally identified using protein-interaction assays with the adenoviral E1A oncoprotein (6). p300 has been implicated in a number of diverse biological functions, including proliferation, cell cycle regulation, apoptosis, differentiation and DNA damage response (7-10). p300 proteins function primarily as histone acetyltransferases (HATs) and as transcription co-factors for a number of nuclear proteins $(11,12)$. Unlike other HATs, which have substrate specificity for histones, p300 is capable of acetylating all four histones. However, the p300/CBP-associated factor (PCAF) is also the first HAT to be discovered in mammalian systems on the basis of its homology to yeast Gcn5p (13). PCAF is known to acetylate various nuclear proteins in addition to histones (9). We previously reported that PCAF promotes cell growth, cellular invasion and cellular resistance to anticancer agents in bladder cancer cells (14). Furthermore, we also reported that p300 is involved in cell growth as well as cisplatin resistance (15). However, it remains unclear how p300 affects cellular resistance to doxorubicin.

In this study, we investigated the functions of p300 with regard to cellular resistance to doxorubicin in bladder cancer. The results revealed that $\mathrm{p} 300$ expression was downregulated in doxorubicin-resistant cells, and that doxorubicin treatment reduced p300 expression. Furthermore, p300 silencing affected resistance to doxorubicin in bladder cancer.

\section{Materials and methods}

Cell culture. The human urothelial cancer lines, KK47 and T24, were cultured in Eagle's minimal essential medium (EMEM), 
which was purchased from Invitrogen (Carlsbad, CA, USA) and supplemented with $10 \%$ fetal bovine serum (FBS). KK47/ADR and T24/ADR cells were established from KK47 and T24 cells, respectively, as described previously $(4,5)$. All cell lines were maintained in a $5 \% \mathrm{CO}_{2}$ atmosphere at $37^{\circ} \mathrm{C}$.

Antibodies. Anti-p300 (sc-585) antibody was purchased from Santa Cruz Biotechnology (Santa Cruz, CA, USA). Anti-Lamin B1 antibody was purchased from Abcam (Cambridge, MA, USA).

Knockdown analysis using siRNAs. Knockdown analyses using siRNAs were performed as described previously (14-16). Briefly, the following double-stranded RNA 25-bp oligonucleotides were commercially generated (Invitrogen): 5'-AUUAUAGGA GAGUUCACCGGGCAGG-3' (sense) and 5'-CCUGCCCGG UGAACUCUCCUAUAAU-3' (antisense) for p300 siRNA \#1; 5'-UAACAGUGACCUCUCCUGACUCAGG-3' (sense) and 5'-CCUGAGUCAGGAGAGGUCACUGUUA-3' (antisense) for p300 siRNA \#2.

Western blot analysis. Western blot analyses were performed as described previously (14-16). The protein concentration of the extracts was quantified using a protein assay kit based on the Bradford method (Bio-Rad, Hercules, CA, USA). Nuclear extracts $(30 \mu \mathrm{g})$ were separated by $4-20 \%$ SDS-PAGE and transferred to polyvinylidene difluoride (PVDF) microporous membranes (GE Healthcare Bio-Science, Piscataway, NJ, USA) using a semi-dry blotter. The blotted membranes were incubated for $1 \mathrm{~h}$ at room temperature with the primary antibodies described above. Membranes were then incubated for $40 \mathrm{~min}$ at room temperature with a peroxidase-conjugated secondary antibody. The bound antibody was visualized using an ECL kit (GE Healthcare Bio-Science) and the membranes were exposed to X-ray film (GE Healthcare Bio-Science).

Cytotoxicity analysis. Cytotoxicity analyses were performed as described previously by Shiota et al (14-16). Briefly, T24 or KK47 cells $\left(2.5 \times 10^{3}\right)$ transfected with $40 \mathrm{nM}$ of the indicated siRNA were seeded into 96-well plates. The following day, the indicated concentrations of doxorubicin were applied. After $48 \mathrm{~h}$, surviving cells were stained with Alamar Blue Assay (TREK Diagnostic systems, Cleveland, OH, USA) for $180 \mathrm{~min}$ at $37^{\circ} \mathrm{C}$. The absorbance was then measured at $570 \mathrm{~nm}$ using a plate reader (ARVOTM MX, Perkin-Elmer Inc., Waltham, MA, USA).

\section{Results}

p300 is downregulated in doxorubicin-resistant bladder cancer cells. First, we investigated the expression level of p300 in these doxorubicin-resistant cells. As shown in Fig. 1, p300 expression was decreased in both doxorubicin-resistant cell lines (KK47/ ADR and T24/ADR cells lines) compared with their parental cell lines (KK47 and T24 cells lines) at the protein level.

Exposure to doxorubicin downregulates p300 expression in bladder cancer cells. Subsequently, we examined p300 expression after exposure to doxorubicin. Human bladder cancer KK47 cells were exposed to $10 \mathrm{nM}$ of doxorubicin for

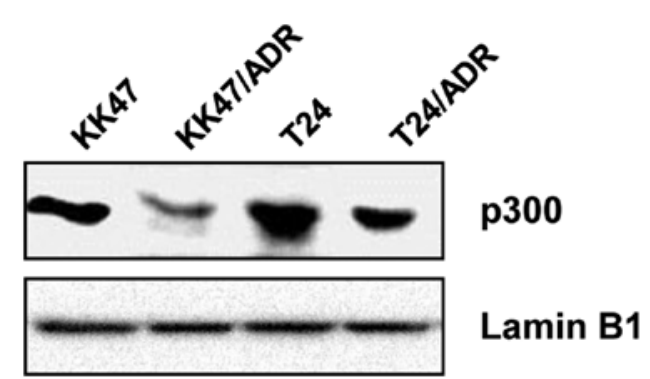

Figure 1. p300 is downregulated in doxorubicin-resistant bladder cancer cells. Nuclear extracts from bladder cancer cells (KK47 and T24 cells) and counterpart doxorubicin-resistant cells (KK47/ADR and T24/ADR cells) were subjected to SDS-PAGE, and Western blot analyses were performed with the indicated antibodies.

$\mathbf{A}$

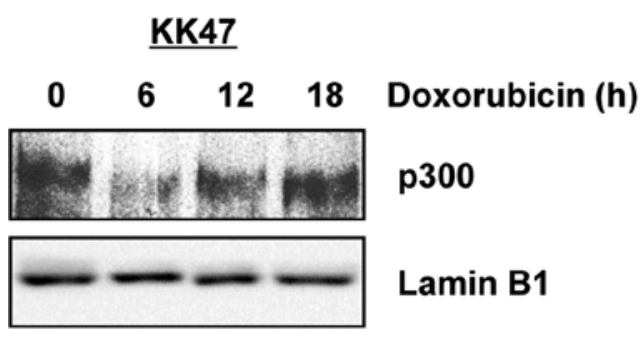

B

$\underline{T 24}$

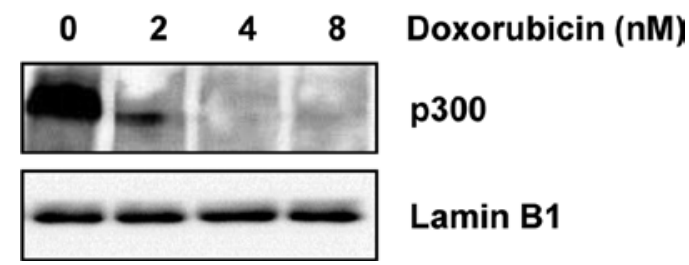

Figure 2. Exposure to doxorubicin downregulates p300 expression in bladder cancer cells. (A) KK47 cells were treated with $10 \mathrm{nM}$ of doxorubicin for the indicated durations. Nuclear extracts were subjected to SDS-PAGE, and Western blot analyses were performed with the indicated antibodies. (B) T24 cells were treated with the indicated concentrations of doxorubicin for $6 \mathrm{~h}$. Nuclear extracts were subjected to SDS-PAGE, and Western blot analyses were performed with the indicated antibodies.

various durations. Western blot analyses showed that $\mathrm{p} 300$ expression level was downregulated by doxorubicin exposure in a peak at $6 \mathrm{~h}$ after doxorubicin exposure (Fig. 2A). Similarly, p300 expression in T24 cells was also reduced by exposure to doxorubicin in a dose-dependent manner (Fig. 2B).

Suppression of p300 renders bladder cancer cells resistant to doxorubicin. Finally, we examined whether p300 affects the cell survival rates after treatment with doxorubicin in KK47 cells. As shown in Fig. 3A, p300 silencing using previously validated p300-specific siRNAs caused KK47 cells to become resistant to doxorubicin. Similarly, cellular resistance to doxorubicin in T24 cells was augmented by p300 shutdown (Fig. 3B) $(15,16)$. 
A

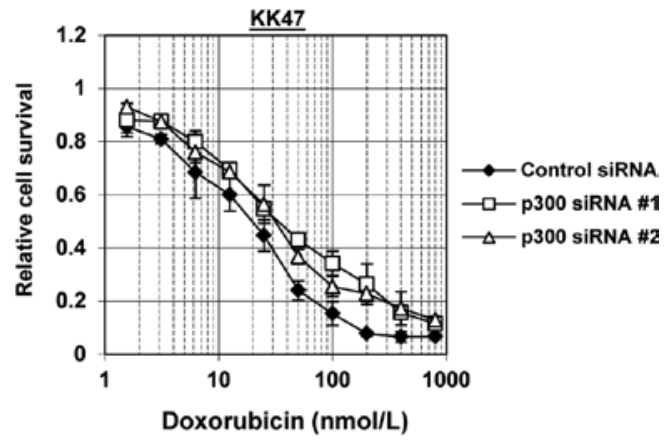

B

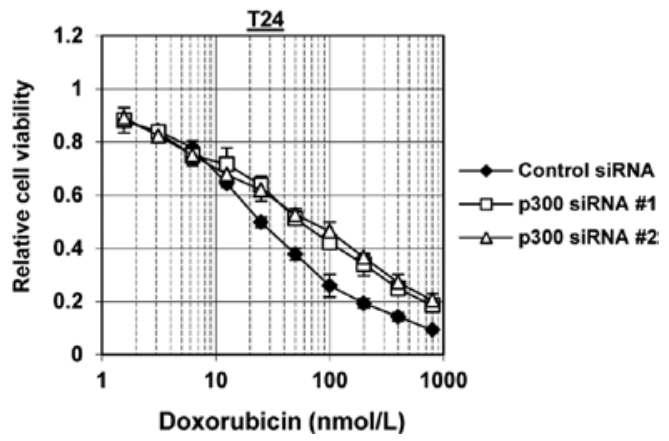

Figure 3. Suppression of p300 renders bladder cancer cells resistant to doxorubicin. KK47 (A) and T24 (B) cells were transfected with $40 \mathrm{nM}$ of the indicated siRNA. On the following day, various concentrations of doxorubicin were added. After $48 \mathrm{~h}$, cell survival rates were analyzed by cytotoxicity analyses. Cell survival in the absence of doxorubicin was set as 1. Data represent the means $\pm \mathrm{SD}$.

\section{Discussion}

In this study, p300 expression was reduced in doxorubicinresistant bladder cancer cells. Although the mechanism of this downregulation remains unclear, it may result from the direct effect of the doxorubicin treatment, as exposure to doxorubicin also reduced p300 expression. Doxorubicin is known to exert oxidative stress in various cell systems $(17,18)$. We previously reported that p300 expression was also suppressed by exposure to cisplatin, which can also exert oxidative stress $(15,19,20)$. Therefore, oxidative stress may be involved in the regulation of p300 expression.

We previously reported that PCAF suppression reduced the expression of YB-1, resulting in retarded cell growth and vulnerability to cisplatin and doxorubicin in bladder cancer cells (14). Inversely, p300 suppression induced YB-1 expression, resulting in augmented cell growth and cellular resistance to cisplatin $(14,16)$. However, the involvement of p300 in doxorubicin resistance remained unknown. This study reveals that p300 suppression renders bladder cancer cells resistant to doxorubicin. This finding is compatible with the result that p300 was downregulated in doxorubicin-resistant cells. p300 may confer resistance to doxorubicin by modulating the expression levels of target genes. YB-1 is known to be associated with doxorubicin resistance (14). We previously showed that YB-1 expression was shown to be affected by p300 in KK47 cells but less so in T24 cells which have a low YB-1 expression (16). p300 affected chemosensitivity to doxorubicin in both cells. Thus, p300 may be involved in drug resistance through molecules other than YB-1 as p300 interacts with a number of proteins that may affect drug sensitivity.

Somatic missense mutations of EP300, the gene encoding p300, which is thought to be a tumor-suppressor gene, occur in a number of malignancies. Chromosome translocations targeting EP300 have been reported in acute myeloid leukemia and treatment-related hematological disorders (21). EP300 gene mutations that result in truncated p300 protein products or amino-acid substitutions in critical protein domains have also been shown in solid tumors (21). Thus, p300 activity may be downregulated in broad range of cancers. Therefore, such cancers with low p300 activity may be resistant to doxorubicin chemotherapy regimens. Hense, the strategy to restore the activity of p300 in such cancers seems to be promising because it is predicted to suppress cell growth as well as augment the chemosensitivity to cisplatin and doxorubicin.

In summary, this study reveals that p300 is downregulated in doxorubicin-resistant bladder cancer cells and by doxorubicin treatment. Consistent with this, p300 suppression promoted drug resistance to doxorubicin in bladder cancer cells. Taken together, this study reveals that p300 may be a promising molecular therapeutic target through modulating chemosensitivity to doxorubicin in bladder cancer.

\section{Acknowledgements}

This study was supported in part by Health Sciences Research Grants for Clinical Research for Evidenced-Based Medicine and Grants-in-Aid for Cancer Research (016) from the Ministry of Health, Labor and Welfare, Japan; Kakenhi grants (22591769) from the Ministry of Education, Culture, Sports, Science, and Technology of Japan (MEXT) and a Grant-in-Aid for Cancer Research from the Fukuoka Foundation for Sound Health, Japan. We would like to thank Ms. Noriko Hakoda and Ms. Seiko Kamori for their technical assistance.

\section{References}

1. Jacobs BL, Lee CT and Montie JE: Bladder cancer in 2010: How far have we come? CA Cancer J Clin 60: 244-272, 2010.

2. Vaishampayan U: Systemic therapy of advanced urothelial cancer. Curr Treat Options Oncol 10: 256-266, 2009.

3. Naito S, Yokomizo A and Koga H: Mechanisms of drug resistance in chemotherapy for urogenital carcinoma. Int J Urol 6 : 427-439, 1999.

4. Kimiya K, Naito S, Soejima T, et al: Establishment and characterization of doxorubicin-resistant human bladder cancer cell line, KK47/ADM. J Urol 148: 441-445, 1992.

5. Hasegawa $\mathrm{S}$, Abe T, Naito $\mathrm{S}$, et al: Expression of multidrug resistance-associated protein (MRP), MDR1 and DNA topoisomerase II in human multidrug-resistant bladder cancer cell lines. Br J Cancer 71: 907-913, 1995.

6. Eckner R, Ewen ME, Newsome D, et al: Molecular cloning and functional analysis of the adenovirus E1A-associated 300-kD protein (p300) reveals a protein with properties of a transcriptional adaptor. Genes Dev 8: 869-884, 1994.

7. Giles RH, Peters DJ and Breuning MH: Conjunction dysfunction: CBP/p300 in human disease. Trends Genet 14: 178-183, 1998.

8. Giordano A and Avantaggiati ML: p300 and CBP: partners for life and death. J Cell Physiol 181: 218-230, 1999.

9. Goodman RH and Smolik S: CBP/p300 in cell growth, transformation, and development. Genes Dev 14: 1553-1577, 2000.

10. Chan HM and La Thangue NB: p300/CBP proteins: HATs for transcriptional bridges and scaffolds. J Cell Sci 114: 2363-2373, 2001.

11. Ogryzko VV, Schiltz RL, Russanova V, et al: The transcriptional coactivators p300 and CBP are histone acetyltransferases. Cell 87: 953-959, 1996. 
12. Ghosh AK and Varga J: The transcriptional coactivator and acetyltransferase p300 in fibroblast biology and fibrosis. J Cell Physiol 213: 663-671, 2007.

13. Yang XJ, Ogryzko VV, Nishikawa J, et al: A p300/CBP-associated factor that competes with the adenoviral oncoprotein E1A. Nature 382: 319-324, 1996.

14. Shiota M, Yokomizo A, Tada Y, et al: P300/CBP-associated factor regulates Y-box binding protein-1 expression and promotes cancer cell growth, cancer invasion and drug resistance. Cancer Sci 101: 1797-1806, 2010.

15. Shiota M, Yokomizo A, Kashiwagi E, et al: Foxo3a expression and acetylation regulate cancer cell growth and sensitivity to cisplatin. Cancer Sci 101: 1177-1185, 2010.

16. Shiota M, Song Y, Yokomizo A, et al: Foxo3a suppression of urothelial cancer invasiveness through Twist1, Y-box-binding protein 1, and E-cadherin regulation. Clin Cancer Res 16: 5654-5663, 2010.
17. Kalyanaraman B, Joseph J, Kalivendi S, et al: Doxorubicininduced apoptosis: Implications in cardiotoxicity. Mol Cell Biochem 234-235: 119-124, 2002.

18. Aluise CD, Sultana R, Tangpong J, et al: Chemo brain (chemo fog) as a potential side effect of doxorubicin administration: Role of cytokine-induced, oxidative/nitrosative stress in cognitive dysfunction. Adv Exp Med Biol 678: 147-156, 2010.

19. Rybak LP, Whitworth CA, Mukherjea D, et al: Mechanisms of cisplatin-induced ototoxicity and prevention. Hear Res 226: 157-167, 2007.

20. Chirino YI and Pedraza-Chaverri J: Role of oxidative and nitrosative stress in cisplatin-induced nephrotoxicity. Exp Toxicol Pathol 61: 223-242, 2009

21. Iyer NG, Ozdag H and Caldas C: p300/CBP and cancer. Oncogene 23: 4225-4231, 2004 